\title{
Investigation of Sound Absorption Properties of Hybrid Multilayer Density Gradient Coir Structure
}

\author{
Vaishnavi Muralidharan ${ }^{1}$ and G Thilagavathi ${ }^{2}$ \\ hod.textile@psgtech.ac.in_vaishnavi4397@gmail.com \\ Department of Textile Technology, PSG College of Technology, Coimbatore, India. ${ }^{1,2}$
}

\begin{abstract}
In this work, hybrid structures from coir nonwoven matts and snippets (finely shredded denim fabric) were developed for determining the sound absorption property. Multilayer density gradient structures were first developed by layering compressed needle punched coir nonwoven matts of increasing densities. The sound absorption behaviour of showed good absorption in the mid and high frequency range because of the increase in density through the thickness that led to better frictional loss of the sound wave. Noise Reduction Coefficient (NRC) of 0.3 was achieved from a gradient density structure comprising of coir fibres alone. Hybrid structure was developed by layering the density gradient coir structure with a sound facing layer (made up of $1 \mathrm{~mm}$ snippets sprayed on the surface of coir sheet). Addition of snippets formed small sized pores on the surface of the coir sheet thus increasing the surface area. The thin panel like structure of the sound facing layer absorbed low frequency showing characteristic peaks but showed decreased absorption in high frequency. Snippets improved vibrational loss and increased low frequency sound absorption achieving NRC of 0.5 .
\end{abstract}

Keywords: Coir fibre; Density Gradient Structure; Cotton snippets Hybrid Structure; Sound Absorption.

\section{Introduction}

Sound is a mechanical energy in the form of longitudinal wave that requires an elastic medium like air for propagation. Sound wave is composed of a spectrum of frequencies. The human hearing frequency range is from $20 \mathrm{~Hz}$ to $20000 \mathrm{~Hz}$. The frequency range is divided into low frequency (below $1000 \mathrm{~Hz}$ ), mid frequency $(1000$ to $2000 \mathrm{~Hz}$ ), and high frequency (above $2000 \mathrm{~Hz}$ ) [1]. Noise is an unwanted and unpleasant sound that is a form of pollution. Environmental noise interferes with the human comfort levels. Absorption of sound is necessary to mitigate the ill effects of noise pollution [2]. Sound absorbers are materials used for noise control during sound propagation.

Coir is a natural fibre that is obtained from the outer shell of coconut. It has the highest amount of lignin content (46-48\%) amongst all natural fibres. High content and low density of lignin create greater inner porosity in the fibre that provides good sound absorption [3]. Tran et al. (2014) have analysed the cross section of a typical coir fibre and have observed that each coir fibre is comprised of many elementary fibres having lumens inside them and has a large hole like a canal termed as lacuna, present in the centre of the fibre running along the axis of the fibre. The presence of lumens and lacuna provide fibre porosity of about 22 to $30 \%$ [4]. 
The inherent porosity and high lignin content are a few suitable properties of coir fibres for the use in sound absorption. It is an ideal material for good sound absorption at high frequency.

A density gradient structure is one in which the density of the material gradually increases through the thickness of the material. In a hybrid structure, more than one type of material is used in forming the structure and each material plays a part in attenuating sound energy. In this research work, sound absorption behaviour of multilayer hybrid coir structure is studied. Porous coir nonwovens are compressed and layered in a density gradient manner for improved sound absorption at mid and high frequencies. The structure is then layered with a hybrid face layer that comprises of coir nonwoven and cotton snippets (finely shredded denim fabric to $1 \mathrm{~mm}$ size) for catering to the low frequency sound waves.

\section{METHODOLOGY}

\subsection{Development of coir matt}

Coir fibres were purchased from local market and needle punched to form a $1000 \mathrm{gsm}$ nonwoven matt at PSG Tech's COE INDUTECH, Coimbatore. The nonwoven matts were about $16 \mathrm{~mm}$ in thickness which were then treated with $20 \%$ latex and cured in a hot press at $110^{\circ} \mathrm{C}$ and 150 ton pressure for 15 minutes for binding the fibres to give good structural integration. The density of the matt was varied by compressing the matts to different thickness during curing. The details of the samples prepared are given in Table 1.

Table 1: Coir matts developed

\begin{tabular}{|c|c|c|c|}
\hline Coir Matt Code & Latex Treatment & Thickness $(\mathrm{mm})$ & Density $\left(\mathrm{kg} / \mathrm{m}^{3}\right)$ \\
\hline PC16 & No & 16 & 62.5 \\
\hline D1 & Yes & 10 & 100 \\
\hline D2 & Yes & 8 & 125 \\
\hline D3 & Yes & 6 & 166.67 \\
\hline
\end{tabular}

\subsection{Development of multilayer structure}

Developed coir nonwovens of different densities were layered in such a manner that the lower density matt faced the sound source and the density increased in the consecutive layers. Two such structures were developed by layering the coir nonwovens. A brief description of the multilayer structures developed are given in Table 2. The sample DG16 was developed by layering $10 \mathrm{~mm}$ and $6 \mathrm{~mm}$ compressed coir nonwoven matts with the $10 \mathrm{~mm}$ layer facing the sound source. The total thickness of the structure was $16 \mathrm{~mm}$. The sound absorption property of this structure was compared with $16 \mathrm{~mm}$ plain coir nonwoven without any latex treatment or hot pressing to evaluate the effect of gradient structure on sound absorption for same thickness materials. This plain coir nonwoven is coded as PC16. The sample DG24 was developed by layering $10 \mathrm{~mm}, 8 \mathrm{~mm}$ and $6 \mathrm{~mm}$ compressed coir nonwoven matts with $10 \mathrm{~mm}$ layer facing the sound source. The total thickness of the structure was $24 \mathrm{~mm}$ with density gradually increasing through the structure.

Apart from these samples, a plain homogeneous coir structure was also formed with no gradient density. The density of the structure was $100 \mathrm{~kg} / \mathrm{m}^{3}$ which was same as the density of 
the face layers of the gradient structures. This structure was developed to compare the effect of gradient density on sound absorption.

Table 2: Multilayer structures developed

\begin{tabular}{|l|l|}
\hline Sample Code & Description \\
\hline DG16 & Coir matts D1 and D3 layered \\
\hline DG24 & Coir matts D1, D2 and D3 layered \\
\hline H24 & 2400gsm coir matt hot pressed to 24mm \\
\hline
\end{tabular}

\subsection{Development of hybrid structure}

Hybrid structure was developed by layering a panel like hybrid face layer with the multilayer gradient structure. The panel like face layer was formed by depositing a thin, even layer of snippets manually using a sieve over the latex sprayed coir matt before curing. Snippets was formed by shredding waste denim fabrics to fine particles of $1 \mathrm{~mm}$ size. The snippet sprayed coir matt was then compressed with full load in the hot press without any thickness liner to form a panel like sheet with snippets adhered to the surface layer forming very small pores. A brief description of the hybrid face layers developed is given in Table 3.

Table 3: Hybrid face layers developed

\begin{tabular}{|c|l|}
\hline $\begin{array}{c}\text { Sample } \\
\text { Code }\end{array}$ & Description \\
\hline $0 \% \mathrm{Sp}$ & Compressed coir sheet without snippets on surface \\
\hline $5 \% \mathrm{Sp}$ & Compressed coir sheet with $5 \%$ snippets by weight of coir matt \\
\hline $10 \% \mathrm{Sp}$ & Compressed coir sheet with 10\% snippets by weight of coir matt \\
\hline
\end{tabular}

The samples are coded for identification with the amount of snippets sprayed on the coir surface. A control panel without snippets was formed to investigate the effect of a face layer and snippets on sound absorption. The hybrid face layer was layered with multilayer structures to form hybrid multilayer structures. Table 4 gives a brief description of the hybrid structures developed.

Table 4: Hybrid structures developed

\begin{tabular}{|l|l|}
\hline Sample Code & Description \\
\hline $0 \%$ DG24 & $0 \%$ Sp layered with DG24 \\
\hline $5 \%$ DG24 & $5 \%$ Sp layered with DG24 \\
\hline $10 \%$ DG24 & $10 \%$ Sp layered with DG24 \\
\hline
\end{tabular}

\subsection{Sound absorption characterization}

The sound absorption behaviour of the developed sample was analyzed using impedance tube method. Noise Reduction Coefficient (NRC) was tested as per ASTM E-1050 2008 [5].

\section{Results And Discussion}

\subsection{Effect of Gradient Structure}

The sound absorption behaviour of raw coir matt (PC16), gradient density structure of $16 \mathrm{~mm}$ (DG16) and gradient density structure of 24mm (DG24) are shown in Figure 1. Plain coir matt (PC16) of $16 \mathrm{~mm}$ thickness without any latex treatment absorbed sound mainly in the 
high frequency. The absorption coefficient increased with increase in frequency. Maximum absorption coefficient of 0.44 was observed at $6400 \mathrm{~Hz}$. The absorption graph showed a gradual increase upto $4000 \mathrm{~Hz}$ and beyond this frequency, the rate of increase in absorption was reduced and attained a saturated maximum value. The plain coir matt showed an NRC of 0.15 with absorption coefficient not crossing over 0.5 even at high frequency. The sound absorption curve of gradient structure (DG16) showed a sharp increase in absorption with frequency from $1000 \mathrm{~Hz}$, reached a maximum absorption of 0.69 at $2500 \mathrm{~Hz}$ beyond which absorption became a constant. Gradient structure showed better sound absorption with NRC of 0.25 for the same thickness, improving sound absorption in both mid and high frequencies because of better frictional loss of sound wave in the structure. The low density face layer allows the sound waves to enter into the structure easily. With the propagation of the sound, due to increased density more frictional loss converts the sound energy to thermal energy. The higher density layer has higher flow resistance with reduced porosity and pore size that increases the tortuosity of the structure. This aids in better sound attenuation [1].

Sound absorption of $24 \mathrm{~mm}$ coir structure with homogeneous density throughout the structure $(\mathrm{H} 24)$ showed a gradual increase in absorption coefficient with increase in frequency with a maximum absorption coefficient of 0.62 at $5000 \mathrm{~Hz}$ and NRC of 0.15 . This structure showed good absorption mainly in the high frequency range. Gradient structure of $24 \mathrm{~mm}$ thickness (DG24) absorbed well in mid and high frequency range with sound absorption coefficient of 0.3 at $1000 \mathrm{~Hz}$ and increased sharply to 0.76 at $2000 \mathrm{~Hz}$, beyond which the increase in sound absorption was very negligible. The maximum absorption coefficient was 0.8 at $4000 \mathrm{~Hz}$ and the NRC was 0.30 . Gradient density structure showed better sound absorption than a homogeneous density structure of the same thickness with almost doubling the NRC value in gradient density structure.

The improved sound absorption of DG24 compared to DG16 was because of the increased thickness of the sample and increased density in the structure. Thicker structure has larger impedance that entraps longer wavelength sound waves. Frequencies being an indirect relationship of wavelength, the low frequency sound waves are intercepted well by the thicker samples and causes better frictional loss [6]. Also, the thicker sample DG24 had an extra layer of coir fibres than the DG16 sample. The extra layer provided more fibres for interaction with the sound waves.

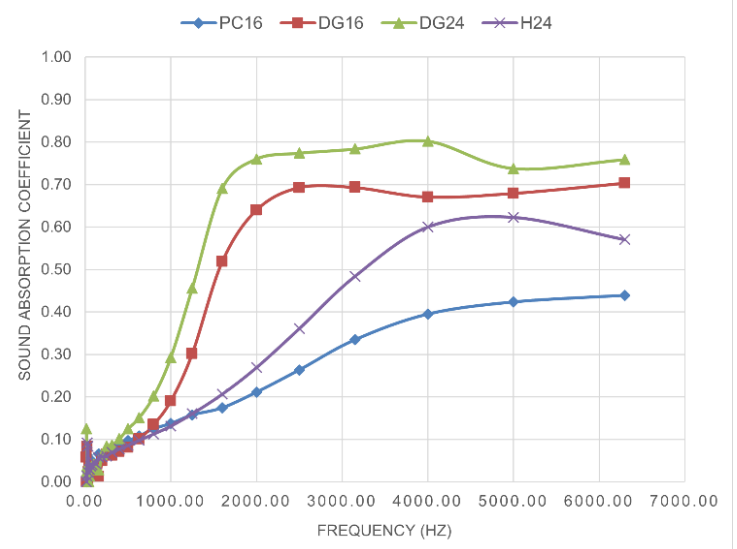

Figure 1: Effect of gradient structure on sound absorption

\subsection{Effect of Snippets}


The sound absorption behavior of coir matt compressed to a sheet $(0 \% \mathrm{Sp})$, coir matt sprayed on the surface with $5 \%$ snippets by weight of coir matt $(5 \% \mathrm{Sp})$ and $10 \%$ snippets by weight of coir matt $(10 \% \mathrm{Sp})$ are shown in Figure 2a to determine the effect of the snippets on the surface and its quantity on sound absorption. The developed face layers resembled a perforated panel with pores in them. The difference being that the pores in these layers were random and not arranged. Sound absorption by the plain coir sheet without snippets $(0 \% \mathrm{Sp})$ showed a gradual increase in frequency upto $4000 \mathrm{~Hz}$ followed by rapid increase until $5000 \mathrm{~Hz}$ beyond which it remained almost constant. The maximum absorption coefficient was 0.51 at $6300 \mathrm{~Hz}$ and NRC was 0.05 . The very low value of sound absorption was because of the thickness of the sample. The sheet was $1.26 \mathrm{~mm}$ in thickness. The absorption at low frequency was very less due to lack of thickness while the high frequency absorption was comparatively higher due to frictional loss with the coir fibres.

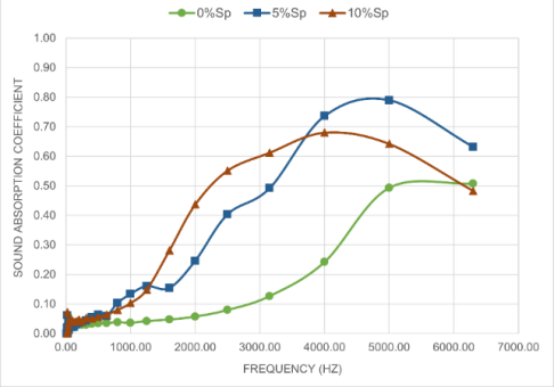

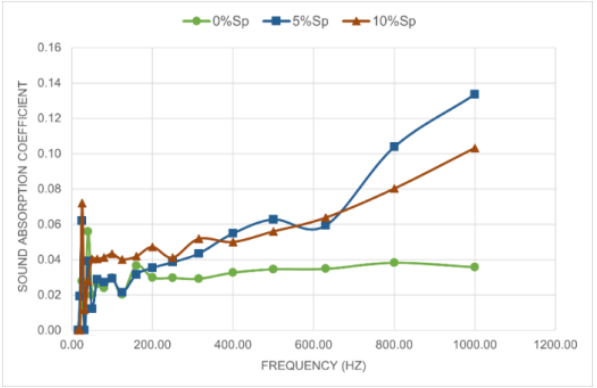

b

Figure2: Effect of snippets on sound absorption: $\mathrm{a}$ - whole frequency range; $\mathrm{b}$ - below

$000 \mathrm{~Hz}$

The sound absorption of $5 \%$ snippets sprayed by weight over the coir nonwoven surface $(5 \% \mathrm{Sp})$ showed a continuous increase in sound absorption upto $5000 \mathrm{~Hz}$ and beyond this frequency the absorption slightly decreased. The maximum sound absorption coefficient was 0.79 at $5000 \mathrm{~Hz}$ and NRC was 0.10 . Compared to the control coir sheet without snippets, NRC was doubled in $5 \% \mathrm{Sp}$. This could be attributed to the even deposition of snippets on the surface that improved the surface area of the face side for better interaction with sound waves, thus increasing lower frequency sound absorption. Similarly, $10 \% \mathrm{Sp}$ sample showed better low frequency sound absorption that increased gradually beyond $1250 \mathrm{~Hz}$ upto $4000 \mathrm{~Hz}$ and slightly decreased thereafter. The maximum absorption coefficient was 0.68 at $4000 \mathrm{~Hz}$ and NRC was 0.15 . Although the maximum absorption coefficient was lesser than $5 \% \mathrm{Sp}$, NRC was higher due to better mid frequency and broad range absorption. This could be attributed to the higher content of snippets on the surface that increased the density and flow resistivity of the surface.

Figure $2 \mathrm{~b}$ shows the sound absorption of the samples in the low frequency range (below $1000 \mathrm{~Hz}$ ) for a better perspective. It is observed that $5 \% \mathrm{Sp}$ performed better in the low frequency range than $10 \% \mathrm{Sp}$. In the $5 \% \mathrm{Sp}$ structure, the snippets on the surface were optimum to produce good vibrational loss of the panel like structure. The $10 \%$ Sp structure had large amount of snippet on the surface that increased the density, blocking the penetration of low frequency waves and could possibly reflect them. Hence 5\% snippets by weight sprayed over the coir sheet surface was optimum to produce an even surface with high surface area and good low frequency absorption by vibrational loss.

\subsection{Effect of Hybrid Face Layer}


The sound absorption behaviour of hybrid structures 0\%DG24, 5\%DG24 and 10\%DG24 are shown in Figure 3a and compared with sound absorption of DG24 to determine the effect of adding a face layer to the gradient structure and the role of snippets in the hybrid structure. Hybrid structure showed improved sound absorption in the lower frequency. Unlike the gradient structure that showed the behaviour of a porous absorber, the face layer in hybrid structure acted as a panel thus showing characteristic resonance peak and the absorption reduced in the high frequency. Hybrid structure 0\%DG24 showed increasing absorption reaching a maximum coefficient of 0.92 at $1600 \mathrm{~Hz}$ beyond which absorption decreased with increase in frequency dropping to about 0.6 at $4000 \mathrm{~Hz}$. The structure showed a typical characteristic peak due to the addition of the face layer that led to resonance in the system. The NRC was 0.50 which is about $66.67 \%$ higher than the gradient structure DG24 without any face layer. Compared to the gradient structure, the hybrid structure absorbed better below $2000 \mathrm{~Hz}$ due to resonance effect that caused vibrational loss of the sound wave. The coir panel being very thin and light could easily vibrate when hit by a sound wave. The minute pores also aided in the process resembling the assembly of a perforated plate with a porous absorber. The drawback was that the absorption beyond $2000 \mathrm{~Hz}$ reduced compared to the gradient structure.

Hybrid structures 5\%DG24 and 10\%DG24 showed a similar absorption pattern as that of $0 \%$ DG24. The maximum sound absorption coefficient of $5 \% \mathrm{DG} 24$ was 0.76 at $1250 \mathrm{~Hz}$ and NRC was 0.50 , while that of $10 \% \mathrm{DG} 24$ was 0.64 at $1000 \mathrm{~Hz}$ and $\mathrm{NRC}$ was 0.45 . Although $10 \% \mathrm{Sp}$ behaved well individually with better NRC, it did not show good result with a porous absorber. The characteristic peak with higher absorption coefficient was achieved with a plain coir sheet because of the light weight of the face layer that could vibrate easier. In the case of snippets sprayed coir sheets, the absorption coefficient at characteristic peaks was lower because of the increased density due to snippets that hindered easy vibrations of the face layer. $5 \%$ DG24 was better than $10 \%$ DG24 because large content of snippets on the surface enhanced reflection of sound waves rather than absorption thus reducing the absorption.

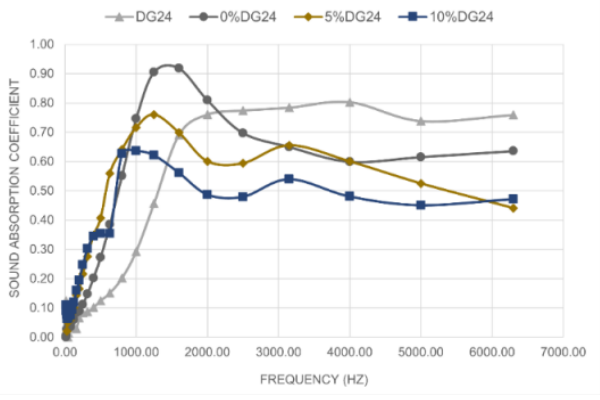

a

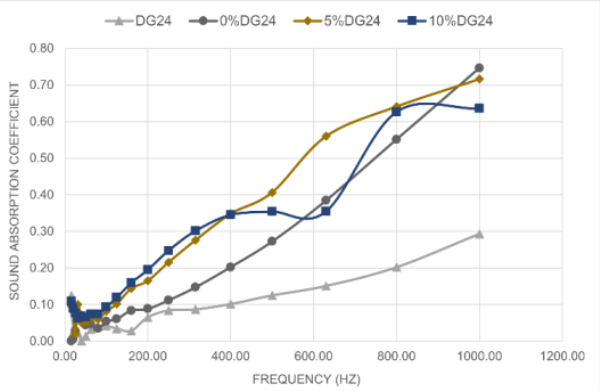

b

Figure3: Effect of hybrid face layer on sound absorption: $a$ - whole frequency range; $b$ below $1000 \mathrm{~Hz}$

Sound absorption behaviour of multilayer structure DG24 and hybrid structures 0\%DG24, $5 \%$ DG24 and $10 \%$ DG24 below $1000 \mathrm{~Hz}$ is shown in Figure $3 \mathrm{~b}$ and it throws light on the low frequency absorption of the hybrid structures. Samples with a face layer performed better in the low frequency than the sample without face layer (DG24). By just introducing a coir sheet facing the sound source, low frequency sound absorption increased sharply due to resonance effect. It was noticed that 5\%DG24 showed increasing sound absorption than the control sample 0\%DG24 and gradient sample DG24. This proved that presence of snippets enhances low frequency sound absorption. This was due to the large surface area and small pores present on the surface that improved the interaction of sound with the surface particles. The 
presence of snippets was expected to increase the flow resistivity and tortuosity of the surface thus increasing frictional loss. This was the reason for the NRC of both 5\%DG24 and $10 \%$ DG24 being same despite the maximum absorption coefficient being smaller for $5 \%$ DG24. The 10\%DG24 sample also showed similar behaviour as that of 5\%DG24 but there occurred drops in absorption patterns. This could be because of the larger density of the face layer that reflected sound waves. Hence it was inferred that 5\% snippets sprayed coir sheet was optimum and showed better broad frequency absorption in a hybrid structure.

\section{Conclusion}

Coir is a natural fibre with suitable sound absorption properties. Coir needle punched matts are used as porous absorber to attenuate high frequency sound waves. Their properties are poor in low frequency. In this research work, results showed that the multilayer structure showed improved sound absorption in both mid and high frequency when compared to a homogeneous layer of the same thickness. The gradient structure was not sufficient for low frequency sound absorption. Hence it was layered with a coir face layer sprayed with snippets on the surface. This sound facing layer acted as a perforated panel showing characteristic peaks by absorbing low frequency sound waves but showed reduced absorption at high frequency. Addition of a snippet layer on the surface of the coir sheet increased the surface area and enhanced the vibrational loss of sound wave by the face layer. An optimum 5\% snippet by weight of a coir matt sprayed on the surface doubled the NRC than plain coir layer. On layering a gradient structure with 5\% snippet sprayed coir matt, there was an improvement of $66.67 \%$ in the NRC. This research work has explored the usage of coir and cotton snippets in a hybrid multilayer density gradient structure for enhanced broad frequency sound absorption. This type of a hybrid structure is an innovative product for the utilization of coir fibres and waste denim fabrics that otherwise would be dumped in landfills. The hybrid structure developed in this work is aimed at achieving best results at minimum possible thickness of the overall structure that is convenient to manufacture.

\section{References}

[1] X. Liu, X. Yan, and H. Zhang, "Effects of pore structure on sound absorption of kapok-based fiber nonwoven fabrics at low frequency,”Text. Res. J.,vol. 86, no. 7, pp. 755-764, 2015.

[2] N. Muthukumar, G. Thilagavathi, S. Neelakrishnan, and P.T. Poovaragan, "Sound and thermal insulation properties of flax/low melt PET needle punched nonwovens,"J. Nat. Fibers,vol. 16, no. 2, pp. 245-252, 2017.

[3] S.K. Saw, K. Akhtar, N. Yadav, and A.K. Singh, "Hybrid composites made from jute/coir fibers: water absorption, thickness swelling, density, morphology and mechanical properties,'J. Nat. Fibers, vol. 11, no. 1, pp. 39-53, 2014.

[4] L.Q.N. Tran, T.M. Nguyen, C.A. Fuentes, T.C. Truong, A.W. Van Vuure, and I. Verpoest, "Investigation of microstructure and tensile properties of porous natural coir fibre for use in composite materials," Ind. Crops Prod.,vol. 65, pp. 437-445, 2015.

[5] ASTM E-1050. 2008. Standard test method for impedance and absorption of acoustical materials using a tube, two microphones and a digital frequency analysis system. West Conshohocken, PA, USA: ASTM Internationals.

[6] G. Thilagavathi, A. Muralikrishnan, N. Muthukumar, S. Neelakrishnanan, in Functional Textiles and Clothing, ed. ByA. Majumdar, D. Gupta, S. Gupta (Springer Nature, Singapore, 2019), p. 37. 
[7] R. Abirami, D.S. Vijayan, Sijo Joseph John, Aldrin Albert, and Alfred Koshy Alex, Experimental Study on Concrete Properties Using Pineapple Leaf Fiber, International Journal of Advanced Research in Engineering and Technology, 11(6), 2020, pp. 913-920, DOI: 10.34218/IJARET.11.6.2020.082 